\title{
Non-Surgical Treatment of Vertigo
}

\author{
P. GHOSH AND M. S. ROHATGI
}

Majority of the cases with dizziness can be treated medically and physiotherapeutically and very few require surgical interventions. In this report one hundred cases of dizziness have been investigated in a busy otolaryngology clinic, diagnosed and treated with drugs and head and neck exercises. The above have been rationalised on a pragmatic basis as to their modes and sites of actions, interactions with various neuropeptides and recalibration of the vestibular subsystem. Above $80 \%$ of the cases were benefited by the regimens laid down in this report. An interestingly significant outcome of this study is that the post-treatment neuro-otological findings have not altered much even in those patients who have improved and resumed their normal activities.

\section{Introduction}

There is constant interaction between the 'phasic' and the 'tonus' element and besides having an afferent system, it has also an effective efferent one, a 'feed-back' and regulating mechanism, that modify both the subjective sensation of vertigo/imbalance and the objective findings (Ghosh \& Kacker, 1979). The complexity of the neurones and the synapses in the vestibular 'subsystem' in the central nervous system with the co-existence of more than one neurotransmitter in a single neurone, multiple pre and postsynaptic receptors, fast and slow postsynaptic potentials, omnopause neurones, reciprocal neuronal units and action at sites distant from that of release of the transmitters enormously multiply the problems of assigning distinct functions to different neurones and neuropeptides. It has been electronystagmographically shown that affection of quick and slowdischarging neuronal units in the paramedian pontine reticular formation are associated with different distinct findings on the electronystatagmograph (Ghosh \& Kacker, 1979 ; Ghosh, 1980 \& 1981). The psychological aspect of the patient complicates it further.

As a clinician, this is a humble attempt at recovering from the

\footnotetext{
P. Ghosh, Additional Professor,

Department of ENT,

All India Institute of Medical Sciences, New Delhi-1 10 029, INDIA,

(formerly, Lecturer in ENT, Faculty of

Medicine, University of Papua New Guinea). M. S. Rohatgi,

Specialist Ear, Nose and Throat Surgeon,

P.O. Box-228, Maitand

N.S. W. 2320, AUSTRALIA.

Reprints request to :

Dr. P. Ghosh,

D-34, Ansari Nagar,

New Delhi-110029

India.
}

'stage of being dysig, meaning stupid or foolish and putting the pragmatic aspect of non-surgical treatment of dizziness on a rational basis as to the sites and modes of actions of different drugs, their modifying effects on the neuropeptides and the effect of head and balance exercises with or without medications.

Affection of the vestibular subsystem, starting from the peripheral labyrinth to the cerebral cortex (temporal lobe and post-central gyrus) leads to dizziness. Nystagmus is the most important manifestation of vestibular disease.

Referring to Fig. 1, one can see $\mathrm{tt}_{i}$ at the fibres from the labyrinth end in the vestibular nuclei and from there they relay in the reticular core, eye nuclei (III, IV and VI), dorsal nucleus of vagus, vomiting centre and chemoreceptor trigger zone, cerebellum (flocculo-nodular lobe and fastigial nuclei) and temporoparietal lobes of cerebral cortex. Some fibres (mossy fibres) go directly from the labyrinth via. the juxtarestiform body to the cerebellum. Conversely, cerebral and cerebellar fibres converge on the vestibular nuclei exerting an inhibitory influence. Damage to these enhances vestibular responses (Ghosh \& Kacker, 1979).

The neurotiansmitters, associated with vestibular mechanism, are noradrenaline, acetylcholine \& Gamma Amino Butyric Acid (Fig. 1). Vestibular receptors are cholinergic. It is likely that a cholinergic transmission to the vestibular neurones cause vertigo. The cholinergic reticulovestibular pathways enhance the activity within the vestibulo-ocular reflex. The mono-aminergic reticulovestibular pathways activated by amphetamine, has an inhibitory in- fluence on the activity of the vestibular nuclei. Thus there are two recticulo-vestibular pathways :

(1) excitatory cholinergic and (2) inhibitory monoaminergic. The distribution of nor-epinephrine in the central nervous system is principally in the hypothalamus and brainstem reticular system (Vogt, 1954). The overall activity depends upon the balance between these transmitters (Eveaton \& Goodhill, 1968). So dizziness could be due to activation of both nor-epinephrine and cholinergic reticular areas. Moreover, the interplay between the quick-discharing and slow-discharging reticular neurones and vestibular nuclei is important (Ghosh and Sen, 1970 ; Ghosh, 1980, 1981). Dizziness would occur when acetylcholine activation exceeds the capacity of nor-epinephrine system. These are also under the regulation of the inhibitory effects of GABA.

Habituation to motion is due to an enhanced response of the norepinephrine mediated system. The increased organisation of the neural system or mobilization of the enzymes necessary for increased norepinephrine activity would be activated by repeated exposure to motion (Wood \& Graybiel, 1970, 1973). This would alter the balance of activity in the central nervous system and prevent motion sickness by allowing nor-epinephrine system to predominate over the acetylcholine system. For the nervous system to recalibrate the relationship between visual, proprioceptive and vestibular signals, repeated head and eye movements are essential. If the level of nor-epinephrine activity is raised by drug; or habituation, or if acetylcholine activity is blocked by drugs, motion sickness may be prevented. 
Material and Methods and observations

One hundred cases of vertigo were studied and managed non-surgically. The aetiologies were as follows :

Group I : Cervical spine trauma sustained 1-2 years back. The age group was between 20-35 years. There were 50 cases.

Group II : Cervical spondylosis : most of the 25 cases were above 45 years.

Group III : Cupulolithiasis-3 cases.

Group IV : Vertebro-basilar insufficiency-9 cases.

Group V : Perilymphatic fistula in the round window-2 cases. Fistulae were closed with fat graft. Mild vertigo persisted for about 3 months and subsequently treated with drugs for 3 months.

Group VI : Undiagnosed : 11 cases. Age range was 45 to 60 years.

Since facilities for conducting electrooculography or optokinetic nystagmography are not available in many institutions, reasonable diagnosis can be and were made by the following :

1. History.

2. Presence and character of spontaneous nystagmus.

3. Kopfschuttel (head-shaking) nystagmus.

4. Unterberger's test (stepping with hands clasped infront).

5. Positional test.

6. Audiometry.

7. Cold caloric test with ice-cold water.

8. X-ray cervical spines, internal auditory meatus and skull, when indicated.

Table I represents the anamnesia, which has been tabulated taking the help of available literature and personal experience, is helpful in differential diagnosis. In quite a good number of cases, history alone could point to the proper diagnosis. In the group with vertebro-basilar insufficiency, the patients had only tran-

PUTATIVE SITES OF ACTION OF ANTIVERTIGINOUS DRUGS

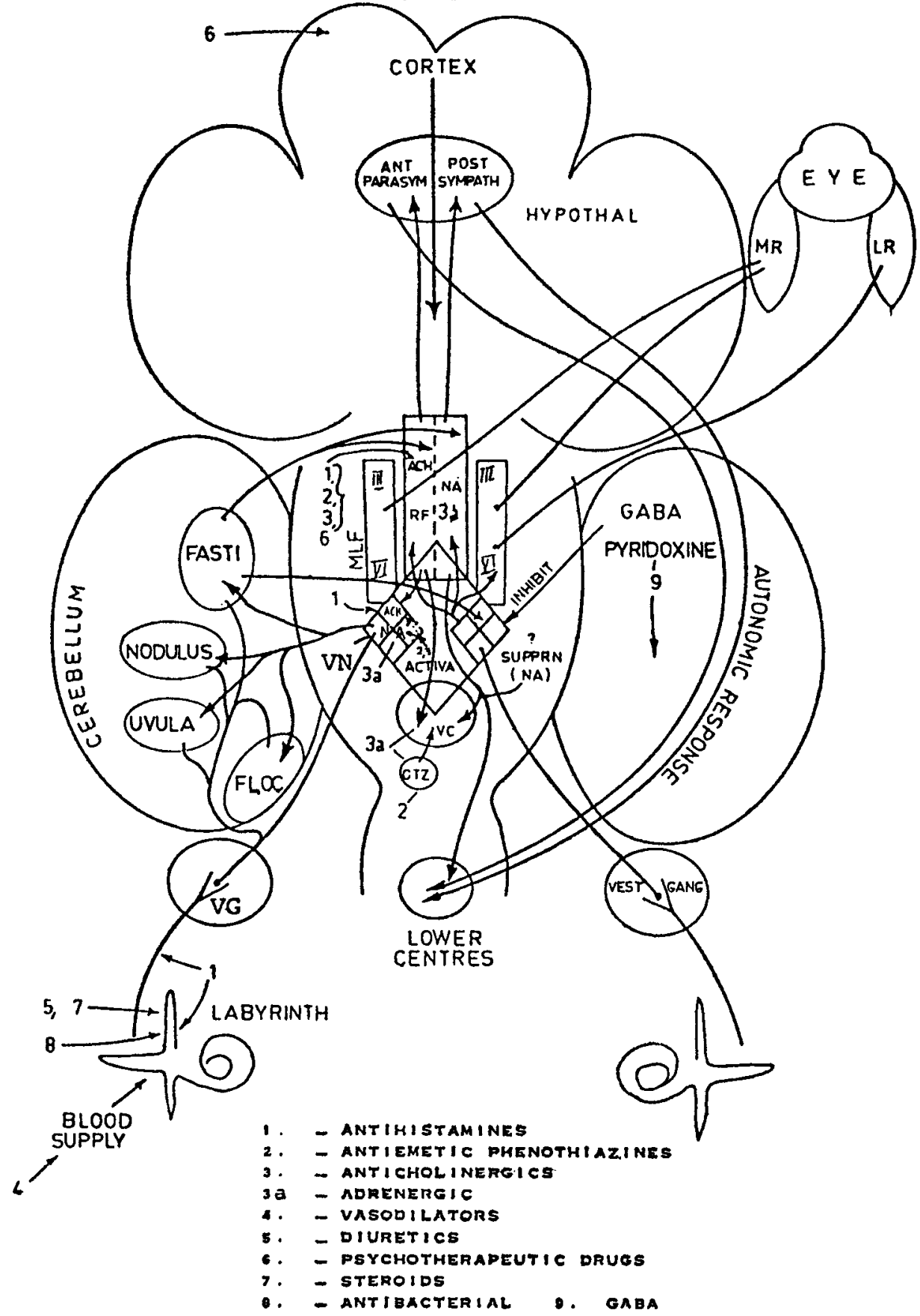

Fig. 1. VG - Vestibular ganglion, VN - Vestibular nuclei, RF - Reticular formation, Supprn - Supperssion, NA - Nor-adrenaline, ACH - Acetylcholine Activa - Activation, CTZ - chemoreceptor Trigger Zone, VC - Vomiting centre, GABA - GammaAmino Butyric Acid, MR - Med, Rectus, LR - Lateral rectus, Ant - Anterior, Post - Posterior, III \& VI-Eye nuclei, Fasti - Fastigious nucleus.

sient numbness and tingling of face, especially in the perioral region. None of the cases showed any neurological deficit.
Spontaneous nystagmus was looked for in sitting and head-erect position. This was present in 5 cases of cervical spine trauma, 2 cases of 
TABLE I

Dizziness (aetiology and site of pathology

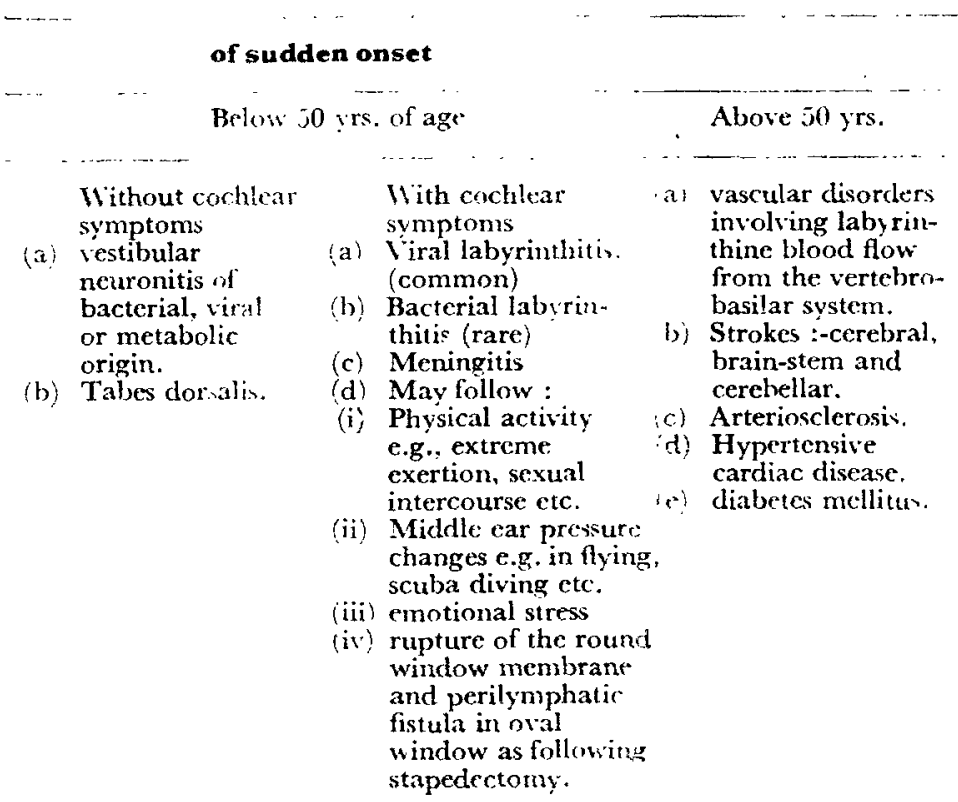

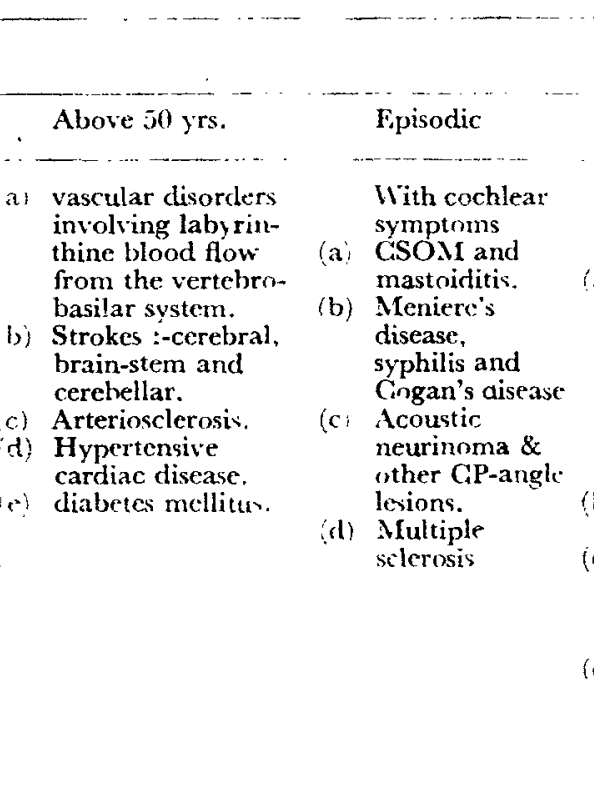

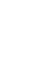

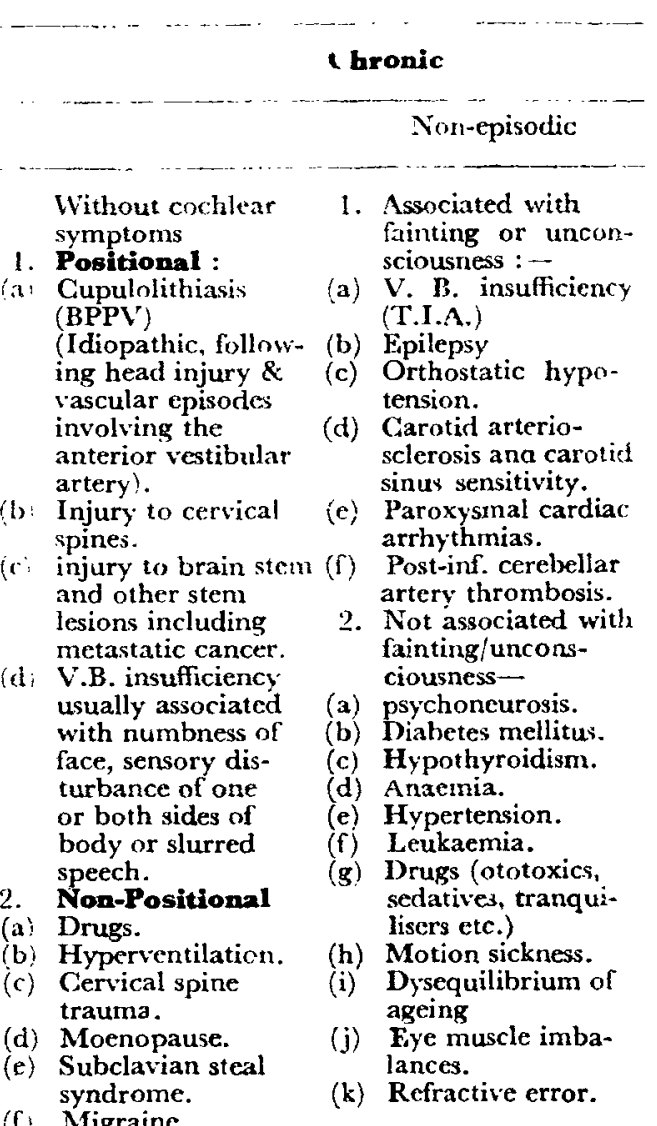

(f) Migraine. cervical spondylosis and 3 cases of vertebro-basilar insuficiency. This was not found to be of any localising value. Two cases of Group IV showed vertical (upwards) nystagmus implicating involvement of both MLFs (Medial Longitudinal Fasciculi). Then the head was (a) tipped backwards, (b) to the right and (c) left, over the shoulders, and (d) forwards for about one minute. Two cases in Group $I$ and 3 cases in Group II showed horizontal rotatory nystagmus fading away within about 15-20 seconds, in $b$ and $c$ positions. The direction of nystagmus was not helpful in localising the lesion. Kopfschuttel nystagmus (on shaking the head sideways around a vertical axis for 20 times) was present in 25 cases with cervical spondylosis (Group II), 2 cases of V.B. insufficiency (Group IV) and 3 of the undiagnosed cases (Group VI). Head shaking produced giddiness similar to the one the patients had been having in 5 of the above cases in Group I ; 2 of Group II ; 2 of Group III ; 2 of Group V and 2 of Group VI (both had Kopfschuttel nystagmus) and dissimilar type in 33 of Group I (including those who had Kopfschuttel nystagmus) and 4 of Group VI, (including those who had Kopfschuttel nystagmus), corroborating the widely accepted impression that the latter is suggestive of central lesion while the former, of peripheral lesion.

Unterberger's test was not of much help in localising the lesion; but it indicated the presence of pathology in the vestibular subsystem. Positional test revealed the following : (a) non-fatiguable nystagmus with direction towards the upper ear was present in 13 cases in Group I (25\% approximately). Out of these, three cases showed decline in nystagmus after fifth positioning and total fatigue on the ninth to tenth ones. The rest showed fatiguable nystagmus towards the undermost ear, (b) in Group II. 10 cases $(40 \%)$ showed non-fatiguable type nystagmu; and out of these, four cases showed decline after the sixth positioning and total decline after the ninth ones, (c) in Group III, all showed fatiguable nystagmus towards the undermost car, (d) in Group IV, five caser (about 50\%) showed dassical non-fatiguable tyfe of nystagmus towards the upper ear, (e) Group V, did not show any positional nystagmus and (f) in Group VI three cases (about 25\%) showed fatiguable type of nystagmus towards the undermost car.

Audiometry was done in all the cuses. 'The following were the positive findings: Groun I-Only 3 cases showed high frequency sensorineural loss. Dynamic ranges between comfortable and uncomfortable levels were not compressed and tone-decay 
test was negative. Group II- $-40 \%$ of them showed high frequency sensori-neural loss in both ears, stggestive of presbyacusis. $30 \%$ of them showed compressed dynamic range, indicative of presence of recruitment. G oup III- Audiometry showed normal hearing. Group IV-8 of them showed high frequency sensori-neural loss and 3 out of them showed bilateral tone decay, indicating neural lesion. Group V-Both cases had high frequency sensorineural loss of 30-40 dB. Group VI-8 cases showed bilateral high frequency sensori-neural loss

\section{CLASS}

\section{A. LABYRINTHINE SUPPRESSANTS}

1. Antihistamines

2. Antiemetics Phenothiazines

3. Anticholinergic

(3) Inhibit Central and Peripheral Cholinergic Pathways, Leading to Blocking of ParaSympathetic System, Depression of Smooth Muscle Activity, Cerebral and medullary Centres.

4. Adrencrgic

(3a) Depress CTZ + VC.

Activate Reti-Vestibular Pathways. Depresses Vest. Nuclei.

5. GABA-Mediated Inhibition

(9) Inhibitory Transmitter.

GABA Fromation Governed by

Pyridoxine-Dependent

Enzymes (Increase in Cerebellum and Brain

Stem). Sodium Valproate Increases

The I,evel of GABA in GNS by

Inhibiting the Enzymatic Break Down of GABA. Good in Head Trauma.

B. VASODH,ATORS

(4) Improve Blood Circulation

(Peripheral and Central). Nicotinic

Acid Reduces Atherosclerosis Also.
1 case showed bilateral hypofunction. Group V-Normal reaction. Group VI-6 were hypofunctioning and 5 normally functioning.

Therapy consisted of drugs and head and neck exercises. The drugs that are used in a case of vertigo can be rationally tabulated (Table-II and Fig.1). The drug;, commonly used in the present study were :

(a) Pyridoxine $40 \mathrm{mg}$ TDS.

(b) Vasodilators : Cyclandelate $200 \mathrm{mg}$. TDS or QID. depending on the intensity of dizziness and

\section{DRUGS}
(a) Cinnarizine
(b) Flunarizine
(c) Dimenhydrinate
$15 \mathrm{mgm} Q \mathrm{GH}$
(d) Promethazine Hydrochlor 25mgm BD
(c) Meclezine Hydrochlor
$25 \mathrm{mgm} \mathrm{BD}$
(f) Astemizole $10 \mathrm{mgm}$ OD

(a) Prochlorperazine 10mgm TDS

(b) Cinnarizine

(c) Promethazine Hydrochlor

(d) Triflupromazine $\mathrm{H}_{y}$ drochlor

(c) Trimeprazine Tartarate

(f) Cyclizine (Marzine)

(a) Atropine 0.4mgm I.M.

(b) Scopalamine 0.6mgm I.M. (Transdermal for Prophylaxis).

(a) d-Amphetamine 10mgm BD

(b) Ephedrine $25 \mathrm{mgm}$ TDS.

(a) Pyridoxine $50 \mathrm{mgm} \mathrm{BD}$

(b) Valproic Acid (Depakene) $15 \mathrm{mgm} / \mathrm{Kg} /$ daily to $30 \mathrm{mgm} / \mathrm{Kg} /$ daily in 3 divided doses. 10mgm. TDS 
CLASS

\section{DIURETICS}

(5) Decrease of Intralabyrinthine Pressure.

Carbonic Anhydrase Inhibitor. Decrease

Reabsorption of $\mathrm{Na}, \mathrm{K}, \mathrm{CL}$ and $-\mathrm{HCO}_{3}$

\section{PSYGHOTROPICS \\ 1. Antianxiety Agents}

2. Antidepressants

E. GORTICOSTEROIDS

\section{F. ANTIBACTERIAL \\ G. CNS-STIMULANTS}

H. CNS-METABOLIC STIMULANT
(7) Decrease Labryinthine Oedema-

Swelling Due To Virus Infection.

Lessen Damage of Acute Viral Labynthitis

if Given Within 24-48 Hrs.

(6) Modify Subjective Response to Vertigo.

Decrease Resting Activity in Vest. Nuclei Diazepam Increases GABA-Mediated Inhibiticn.

Affect Brain Amine Levels by (Tricyclic Antidepressants)

(8) Combat Infection

Cortex \& Reticular Activity

Uptake \& Utilisation of Glucose \& $0_{2}$ increased in Cortex \& Brainstem. Good after Head Trauma. Reticular Facilitatory system.

\section{DRUGS}

(f) Papaverine 150mgm Capsule BD (.5 $\mathrm{mgm} / \mathrm{Kgm}$ Body Wt.)

(g) Duvadilan Retard (Isoxsuprine) $40 \mathrm{mgm}$ BD.

(h) Nicotinic Acid (75mgm TDAC).

(i) Perdilatal (Buphemine Hydrochlor) 6mgm Tab. 2TDS

(j) Thymoxamine $10 \mathrm{mgm} \mathrm{I.V.} \mathrm{or} \mathrm{40-100}$ mgin Orally.

(k) Caffeine $150-250 \mathrm{mgm}$ TDS.

(1) Naftridofuryl (Praxiene) $100 \mathrm{mgm}$ TDS.

(a) Acetazolamide $250 \mathrm{mgm}$ OD For 2 Days in every 3 days.

(b) Hydrochlorthiazide 25mgm 12 Hrly. (K Supplemented if Used for a Long Time. 20 M.Mol/Day)

(a) Diazepam 2-5mgm 8 Hourly.

(b) Phenobarb 15-45 mgm 8 Hourly.

(b) Nortriptyline 10mgm TDS.

Prednisolone 25-100mgm OD. Dexamethasone

Antibiotics

Xanthine Derivatives e.g. Caffeine-150-250 mgm TDS.

Pyritinol 300-600 mgm daily

Good Combinations :

(1) Scopalamine + Dextroamphctamine--Strongest Antivertiginous Combination.

$.6 \mathrm{mgm}$

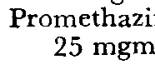
$25 \mathrm{mgm}$

$$
\begin{gathered}
\\
+\quad 10 \text { mgm BD } \\
+ \\
+\quad \text { Ephedrine } \\
25 \text { mgm TDS. }
\end{gathered}
$$

\section{Keywards}

CTZ -. Chemoreceptor Trigger Zone.

VC -- Vomiting Centre

CNS - Central Nervous System.

(c) pyritinol 300-600 mg. daily. Cinnarizine $25 \mathrm{mg}$. TDS was tried on 10 cases. The above were administered for two to three months. Acute exacerbations during the treatment period were managed by dimenhydrinate, prochlorperazine, and tranquilizers for a few days, depending on the responses. Head and Neck exercises were prescribed for six months or more to all the patients. Initially about $70 \%$ of the patients complain of some dizziness on performing the exercises; but they tolerate them subsequently with pleasure without any induced vertigo. Two patients had even vomiting initially and had to discontinue the manoeuvres.

No psychotropic drugs viz. diazepam, was administered routinely since in our experience we found that this interferes with and delays the recalibrating or learning process that takes place in the reticular/centrencephalic system during the above exercises. (Ghosh and Kacker, 1979).

The results of treatment were quite encouraging and are as follows :

Group I : 40 of the cases were back to normal activities and did not complain of dizziness; 5 cases showed improvement and the rest 5 continued to have symptoms as before, Group II : Out of 25 cases, 20 cases showed remarkable improvement. The remaining 5 cases, had improvement to the extent that they could lead reasonably normal life.

Group III : All of them become normal.

Group IV : 6 of them improved and became reasonably active. 3 did not show much improvement though they felt somewhat better. 
Group V: Both the cases became normal.

Group VI : 9 of them improved very well and are leading normal life. The remaining two maintained status quo.

\section{Post-treatment neuro-otological findings :}

Spontaneous horizontal - rotary nystagmus, where present earlier, disappeared in $70 \%$ of the cases. But vertical nystagmus, noted in Group IV cases, persisted though symptomatically they improved remarkably. Initially and later they did not have any neurological deficit. In about $25 \%$ of the cases Kopfschuttel nystagmus disappeared. In rest of the cases, though the"e was subjective improvement, Kopfschuttel nystagmus persisted. Patients withboth similar and dissimilar types of induced vertigo, following headshaking, benefited i.e. there was no difference in their responses to the treatment between the groups with peripheral and central lesions. Similar was the behaviour with regards to fatiguable and non-fatiguable nystagmus on positioning. Both varieties persisted in all the cases, even in those who had subjective improvement. The significant observation is that the neuro-otological findings do

\section{References}

1. Avery, G. S. (Editor), 1980 : Principle and Practice of Clinical Pharmacology and Therapeutics, Sydney and New York, Adis Press, 349-354.

2. Eveaton, A. and Goodhill, V. 1968 : Comparative sequential studies of effects of drugs on the vestibular system of laboratory animals, Acta Otolaryngologica 237 (Suppl) : $1: 26$

3. Ghosh, P. and Sen, D. K., 1970 : Nysta. gmus and Brain lesions, Neurology, India, 18 : 236-240. not show any remarkable change after non-surgical treatments, ven though there is remarkable subjective improvement.

\section{Discussion}

From the present study it is obvious that the lesions in patients with dizziness quite often cannot be precisely localised, though one may have reasonable idea as to their being peripheral or central (suprasegmental) in nature. Nevertheless, disturbances eg. upset homeostasis in the blood circulation and neurotransmitters in the vestibular 'subsystem', incoming abnormal afferent impulses from the periphery (as in cervical spondylosis) to this subsystem and cerebellovestibular incoordination etc. can be effectively managed by non-surgical treatment. The drugs and their rationalised usages are legion (Fig. 1 and Table II); but one can choose a few and treat the patients with gratifying results. The modes of action have been tabulated for ready reference (Table-II) and the drugs can be safely instituted. The rationale behind head and neck exercises lie in: i) recalibrating the functional units in the central nervous. system and ii) enhanced responses in the non-

4. Ghosh, P. and Kacker, S. K., 1979 : Vestibular recruitment and decruitment, Acta Otolaryngologica, 88: 227-234.

5. Ghosh, P. 1980 : Inclination of eyetracking tracing, Journal of Laryngology and Otology 94 : $719-735$.

6. Ghosh, P. 1981 : Round peaked nystagmus, Indian 7 . Otolaryngology 33 : 55-58. epinephrine mediated system as a result of execrise. 'The former is substantiated by the fact that tranquilisers reduce and delay this process by depressing the reticular/centerencephalic system and hence we do not use these drugs while the patient is undergoing exercises (Ghosh and Kacker, 1979). Any patient complaining of dizziness should not be indiscriminately treated with Stemetil, Diligan, Valium etc. as a blanket cover, since this practice is not only not helpful in many cases; but contrarily, it does harm to many in the form of delaying the recalibration process and at times perpetuating the symptoms. Pyridoxin enhances the enzymatic reactions that increase the concentration of GABA which inhibits the vestibular subsystem leading to subjective improvement. Pyritinol increases the uptake and utilisation of glucose and oxygen in the cerebral cortex and brainstem and is very good for head trauma. Vasodilators bring in more nutrition to the cell of the peripheral and central vestibular apparatus. Cinnarizine inhibits the influx of calcium-ions into the vestibular cells, thus, dampening the physiological link in excitation. It also improves the blood flow to the vestibular structures, both peripheral and central.

7. Vogt, M. (1954): The concentration of sympthin in different parts of the nervous system, F. Physiology 123 : 451-481.

8. Wood, C. D. and Graybiel, A., 1970 : A Theory of motion sickness, based on pharmacological reaction, Clinical Pharmacology' and Therapeutics 11 : 621-629

9. Wood, G. D. and Graybiel, A., 1973 : A Theory of motion sickness, Otolaryngologic Clinic of North America, 6 308-629. 